http://jmscr.igmpublication.org/home/

ISSN (e)-2347-176x ISSN (p) 2455-0450

crossref DOI: https://dx.doi.org/10.18535/jmscr/v7i9.106

Journal Of Medical Science And Clinical Research

IGM Publication

An Official Publication of IGM Publication

\title{
Correlation of serum uric acid with lipid profile in pre-eclamptic patients
}

\author{
Authors \\ Lata Bishnoi $^{1}$, Nagraj Soni ${ }^{2 *}$, Anita Verma ${ }^{3}$ \\ ${ }^{1}$ Asst. Professor, ${ }^{2} \mathrm{PhD}$ Scholar, ${ }^{3}$ Asso. Professor \\ Department of Biochemistry, S.P. Medical College, Bikaner, Rajasthan, India \\ *Corresponding Author \\ Nagraj Soni
}

\begin{abstract}
Introduction: Pre-eclampsia is one of the largest causes of maternal and fetal mortality and morbidity. Dyslipidemia and hyperuricemia have been shown to play a significant role in the pathogenesis of the disease and often precede clinical manifestations.

Objective: The aim of the present study was to evaluate the lipid profile among patients who present preeclampsia and correlate these parameters with uric acid.

Materials and Methods: The present study was conducted on 50 normal pregnant women (Group-I), 50 Preeclamptic pregnant women (Group-II). The blood samples collected from all the women and analyzed for serum lipid profile and uric acid.

Results: Mean serum uric acid and triglyceride levels ( $p<0.001$ and $p<0.05$ respectively) were significantly higher and total cholesterol and $L D L-C$ were increased and $H D L-C$ was decreased but the results were not statistically significant in pre-eclampsia group as compared to normal pregnant group. Positive correlation of uric acid was seen with total cholesterol and HDL-C and negative correlation with triglycerides and LDL-C. But the correlation was not statistically significant.

Conclusion: Measurement of serum lipids and uric acid may be helpful in early diagnosis and prevention of maternal and fetal complications in preeclampsia.

Keywords: Preeclampsia, Normal pregnancy, Dyslipidemia, lipid profile, uric acid.
\end{abstract}

\section{Introduction}

Preeclampsia is a pregnancy specific, multisystem disorder characterized by hypertension to an extent of 140/90 mmHg or more accompanied by proteinuria ( $>300 \mathrm{mg} /$ day), edema or both ${ }^{[1]}$. It is one of the most common complications of pregnancy and is a leading cause of maternal and fetal morbidity and mortality ${ }^{[2]}$. Preeclampsia occurs in $7-10 \%$ of pregnancies worldwide. In India the incidence is reported to be $8-10 \%$ of the pregnancies. Preeclemptic disorder is mediated by placental products that reach the maternal circulation and trigger endothelial dysfunction, thereby evoking cardiovascular diseases, such as vasospasm, increased endothelial permeability and activation of thrombogenic mechanisms, and leading to the early events of atherosclerosis. Susceptibility to preeclampsia is also modulated by maternal factors. Hyperuricemia is a common finding in preeclamptic pregnancies. Abnormal renal function, increased tissue breakdown, acidosis and increased activity of the enzyme xanthine oxidase/dehydrogenase may be the reason for elevated serum levels of uric acid in women with preeclampsia ${ }^{[3]}$. Association of alteration of serum lipid profile in essential hypertension is well documented. Abnormal lipid profile is strongly associated with atherosclerotic 
cardiovascular diseases and has a direct effect on endothelial dysfunction. Most important feature in toxemia of pregnancy is hypertension which is supposed to be due to the vasospastic phenomenon in the kidney, uterus, placenta, and brain $^{[4]}$. In the present study we evaluated, correlation of serum uric acid with lipid profile in pre-eclamptic patients.

\section{Materials and Methods}

The present study was carried out in the Department of Biochemistry in collaboration with the Department of Obstetrics and Gynecology of S P Medical College \& PBM hospital, Bikaner. A total number of 100 participants of ages between 16-35 years and gestational age between 20-38 weeks were chosen and they were divided into 2 groups, Group-I : Normal pregnant women $(n=50)$ and Group-II: Pre-eclamptic women $(n=50)$. Women with concomitant disease such as diabetes or a history of gestational diabetes, chronic hypertension , and kidney disease or coagulation were excluded. All pregnant women were taken in the third trimester. Informed consent was taken from all individuals included in the study. Fasting blood samples $(5 \mathrm{ml})$ were collected by venipuncture and analyzed for total cholesterol (TC), serum triglycerides (TGs), high-density lipoprotein-cholesterol (HDL-C) and uric acid were estimated in the both groups. The serum lipid profile was estimated by the enzymatic CHOD-POD method for TC, by the GPO-PAP method for TG and by the CHOD-POD/ Phosphotungstate method for HDL-C. Calculated of VLDL-C and LDL-C using Friedewald's formula (Friedwald et.al.1972). serum uric acid was estimated by uricase method. The estimations carried out of semi autoanalyzer. Data were statistically analyzed by unpaired $t$ test and expressed in terms of $\mathrm{P}$ value $(\mathrm{p}<0.05$, considered).

\section{Results}

Control and pre-eclampsia groups were comparable with respect to age $(21.86 \pm 3.12 \&$ $22.86 \pm 2.80)$ and gestational age $(35.04 \pm 2.43 \&$ $34.44 \pm 2.27)$, respectively. Systolic and diastolic BP were statistically significant $(<0.0001)$ in the pre-eclamptic group compared to normotensive pregnant women.

Serum uric acid, triglycerides and VLDL-C were significantly increased $(\mathrm{p}<0.0001)$ and HDL-C was significantly decreased in pre-eclampsia group compared to control group. Total cholesterol and LDL-C were increased in preeclampsia group as compared to normal pregnant group but the results were not statistically significant (Graph:1).

When uric acid was correlated with lipid fractions (Table:1), positive correlation of uric acid was seen with total cholesterol and HDL-C and negative correlation with triglycerides and LDLC. But the correlation was not statistically significant.

Graph: 1 Comparison of serum lipids \& UA in both groups.

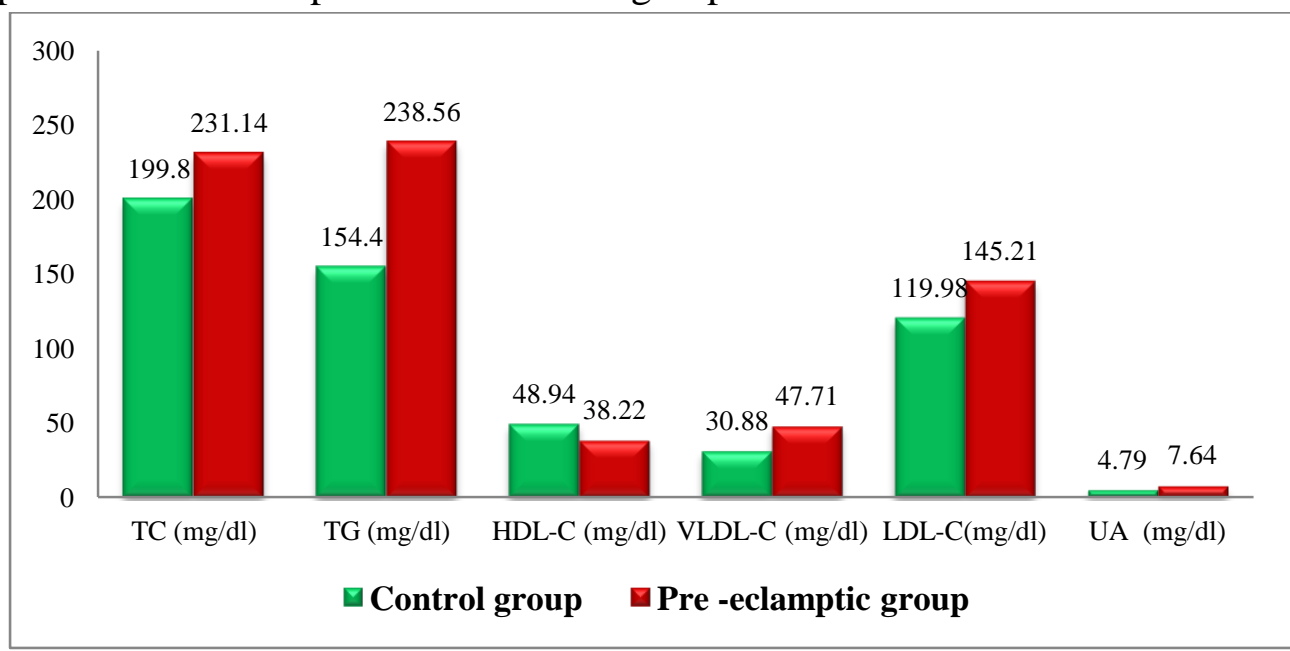


Table 1: Correlation of Serum Uric acid with lipid parameters

\begin{tabular}{|c|c|c|c|}
\hline \multirow{4}{*}{$\begin{array}{c}\text { UA } \\
\text { (mg/dl) }\end{array}$} & Parameters & r-value & P value \\
\cline { 2 - 4 } & $\mathrm{TC}(\mathrm{mg} / \mathrm{dl})$ & 0.051 & 0.50 \\
\cline { 2 - 4 } & $\mathrm{TG}(\mathrm{mg} / \mathrm{dl})$ & -0.030 & 0.61 \\
\cline { 2 - 4 } & HDL-C $(\mathrm{mg} / \mathrm{dl})$ & 0.210 & 0.22 \\
\cline { 2 - 4 } & VLDL-C $(\mathrm{mg} / \mathrm{dl})$ & -0.006 & 0.40 \\
\cline { 2 - 4 } & LDL-C $(\mathrm{mg} / \mathrm{dl})$ & -0.015 & 0.24 \\
\hline
\end{tabular}

$\mathrm{P}<0.05=$ Significant .

\section{Discussion}

Pre-eclampsia usually begins after 20 weeks of pregnancy in a women whose blood pressure had been normal. It can lead to serious, even fatal complications for both mother and baby. Serum triglycerides concentration rise more significantly in pre-eclampsia in our study which corroborated with the findings of many studies ${ }^{[5,6]}$. The major modulator of this hypertriglyceridemia is estrogen as pregnancy is linked with hyperestrogenemia. Hypertriglyceridemia may be linked to hypercoagulability.

We also found increased total cholesterol and LDL-C levels in preeclampsia. Our finding according to previous studies ${ }^{[2,7]}$. Hyperlipidemia during preeclampsia is transient, therefore its pathological role in these women have been ignored. Hypoestrogenemia, seen in preeclampsia, leads to decreased expression of VLDL/apoE receptors resulting in reduced transport of VLDL to fetal compartment and so there is maternal hypertriglyceridemia ${ }^{[7]}$. Elevated triglycerides may compromise vascular functions in several ways. For example, triglyceride rich lipoprotein has a prothrombotic effect ${ }^{[2]}$. We found decrease in serum HDL-C concentration compared to normal pregnant women. This is in consistency with the results of several other studies. Gohil J.T et al demonstrated significant fall in HDL-C in preeclampsia than in non-pregnant and normal pregnant women ${ }^{[2]}$. Increased triglycerides play a role in increased atherogenic small dense LDL and reduced $\mathrm{HDL}^{[7,8]}$. A low level of HDL-C hinders reverse cholesterol transport, which may be a reason for the atherosclerosis like features in preeclampsia mentioned in some studies ${ }^{[2]}$. In the present study, we also found increased serum uric acid level in preeclamptic women which was statistically significant. Our result is in agreement with other studies ${ }^{[1,3,9]}$. Uric acid, an end product of purine catabolism catalyzed by xanthine oxidase, is filtered, reabsorbed and secreted by the kidney. In preeclampsia, glomerular endothelial lesions lead to diminished renal blood flow and glomerular filtration rate and also impaired tubular reabsorption. Hyperuricemia in preeclampsia is thus primarily due to decreased renal clearance and increased tubular reabsorption, because of the reduction in glomerular filtration rate ${ }^{[1]}$.

\section{Conclusion}

Results of our study add to the existing evidence to support pathophysiological role of both dyslipidemia and hyperuricemiain the clinical manifestations of preeclampsia. Measurement of serum lipids and uric acid may be helpful in early diagnosis and prevention of maternal and fetal complications in preeclampsia.

\section{References}

1. Anjum Sayyed and Alka Sontakke, Study of lipid Peroxidation and antioxidant status in Preeclampsia, JKIMSU, 2(2), 2013, 6976.

2. Gohil J.T, Patel P.K and Gupta Priyanka, Estimation of Lipid Profile in subjects of Preeclampsia, The Journal of Obstetrics and Gynecology of India , 61(4), 2011, 399-403.

3. S. A. Bainbridge and J. M. Roberts, Uric Acid as a Pathogenic Factor in Preeclampsia, Placenta, 22, 2008, S67S72.

4. Lindheimer MD, Cunningham FG, Roberts JM. Introduction, History, Controversies, and Definitions in Chesley's Hypertensive Disorders in Pregnancy. Amsterdam, Netherlands: Elsevier; 2009. p. 1- 24.

5. Weerasekera DS, Peiris H. The significance of serum uric acid, creatinine and urinary microprotein levels in 
predicting pre-eclampsia. J Obstet Gynecol 2003; 23:17-9.

6. Anil Bargale, JayashreeV.Ganu, Dhiraj J. Trivedi, Nitin Nagane, Rakesh Mudaraddi and AparnaSagare, Serum Hs-CRP and Uric Acid as Indicator of Severity in Preeclampsia, International Journal of Pharma and Biosciences, 2(3), 2011, B 340-B344.

7. Dr. P. Josephine Latha and Dr. S.Ganesan, Evaluation of Serum Uric Acid and Lipid Profile in Gestational Hypertension, Int J Pharm Bio Sci, 4(2), 2013, 496-502.

8. Rubina Aziz and Tabassum Mahboob, Preeclampsia and Lipid Profile,Pak J Med Sci, 23(5), 2007, 751-754.

9. T. Sunita, K.Sameera and G. Umaramani, Study of Biochemical changes in Preeclamptic women, Int J Biol Med Res, 3(3), 2012,2025-2028. 